\title{
ON FINITELY GENERATED FLAT MODULES
}

\author{
BY \\ WOLMER V. VASCONCELOS
}

Introduction. The aim of this paper is to study conditions which reflect the projectivity of a given finitely generated flat module over a commutative ring. The use of the invariant factors of a module (see below for definition) are very appropriate here: By translating the description by Bourbaki [4] of finitely generated projective modules, one can state that projectivity = flatness + finitely generated invariant factors. Since the invariant factors of a flat module are very peculiar (locally they are either (1) or (0)), the presence of almost any other condition on the module precipitates their finite generation. For instance, consider the following two statements. Let $M$ be a finitely generated flat mocule over the commutative ring $R$ : (i) Let $S$ be a multiplicative set in $R$ consisting of nonzero divisors such that $M_{S}$ (localization of $M$ with respect to $S$ ) is $R_{S}$-projective; then $M$ is projective. (ii) Let $J$ be the Jacobson radical of $R$ and assume that $M / J M$ is $R / J$-projective; then $M$ is projective. The first is a result by Endo [8] who uses homological algebra in the proof, while the second can be viewed as a generalization of the well-known fact that over a local ring a finitely generated flat module is free.

Next we apply these ideas for a look at finitely generated flat ideals. Even though they are not always projective (see below for an example of a principal flat ideal which is not projective) it can be shown this to be the case when the flat ideal is a finite intersection of primary ideals. The criterion mentioned above says that for a finitely generated flat ideal, projectivity is the same as having finitely generated annihilator. For rings with the weakened form of coherency that finitely generated ideals have finitely generated annihilators, one can even show that any finitely generated flat submodule of a projective module is projective.

When used to study the prime ideals of a ring $R$ of weak dimension one we arrive at the fact that a finitely generated prime ideal is either maximal or generated by an idempotent. It is also shown that if every principal ideal is projective then $R$ is semihereditary; if moreover every cyclic flat module is projective, then $R$ is a direct sum of finitely many Prüfer domains.

1. Flatness and projectivity. Throughout $R$ will denote a commutative ring and $M$ a finitely generated $R$-module. We will assume from the reader familiarity with the notions of localization and exterior product, of which repeated use shall be made (see [2], [4] and [7]). For each prime ideal $P$, i.e. for each point of Spec $R$, let $r_{M}(P)$ denote the minimum number of generators of the localization $M_{P}$ over

Received by the editors May 27, 1968. 
$R_{P}$. Thus $r_{M}$ defines an integral valued function on $\operatorname{Spec} R$, sometimes called the rank function of $M$. Bourbaki [4, Chapter II, Theorem 1] characterizes a finitely generated projective module $M$ as being free at each localization $M_{P}$ and such that its rank function is locally constant, Spec $R$ endowed with the usual topology. On the other hand, the $p$ th invariant factor of $M, I_{p}(M)$, is defined as the annihilator of $\bigwedge^{p} M$, the $p$ th exterior power of $M$. It then follows that $r_{M}(P) \geqq p$ precisely for those primes $P \supset I_{p}(M)$, i.e. for $P$ in the closed set $V\left(I_{p}(M)\right)$. Thus $V\left(I_{p}(M)\right)$ $-V\left(I_{p+1}(M)\right)$ is the set of points where the rank of $M$ is exactly $p$. Assume from here on that $M$ is flat; according to [4, p. 167] this is the same as $M$ being free at each prime. As $M$ is finitely generated, $V\left(I_{p}(M)\right)$ is empty for large $p$. Coupled with this the Bourbaki criterion assumes the form: $M$ is projective iff $M$ is flat and $V\left(I_{p}(M)\right)$ is open for all $p$ 's. On the other hand, since $\wedge^{p} M$ is finitely generated, for any localization $R_{P}, I_{p}\left(M_{p}\right)=I_{p}(M) \otimes R_{P}$. But $\wedge^{p} M$ is locally free as localization and formation of exterior powers commute and we conclude that for each prime $P, I_{p}\left(M_{P}\right)$ is either (1) or (0), i.e. the whole localization or the null ideal. In particular $\left(I_{p}(M)\right)^{2}=I_{p}(M)$.

We pause to discuss this condition briefly. Let $I$ be an ideal in a commutative ring $R$ and $M$ a finitely generated $R$-module. If $M=I M$, by writing each element in a generating set of $M$ as a linear combination in all the generators with coefficients in $I$, one gets, via the so-called "determinant trick" [17, p. 255] that there exists $a \in I$ with $(1+a) M=(0)$. One consequence is

Proposition 1.1. Let I be a finitely generated ideal in a commutative ring. Then $I=I^{2}$ iff $I$ is generated by an idempotent.

Also, free of charge, one has

Proposition 1.2. Let $M$ be a finitely generated module over the commutative ring $R$. Then any endomorphism of $M$ onto itself is an automorphism.

Proof. Let $f$ be the given endomorphism of $M$. One can consider $M$ as a module over $R[x]$, where the action of the indeterminate is given by $x m=f(m)$, for $m \in M$. Then $M$ is a finitely generated $R[x]$-module with $M=x M$. By the remark, $(1+s x) M$ $=(0)$ for some $s \in R[x]$. It is then clear that $f$ is a monomorphism.

Going back to the flat module $M$, its invariant factors are finitely generated iff they are generated by idempotents and so $V\left(I_{p}(M)\right)$ is open for all $p$ 's. We can now state

Proposition 1.3. Let $R$ be a commutative ring and $M$ a finitely generated flat module over $R$. Then $M$ is projective iff its invariant factors are finitely generated.

For the portion still to be proved we just recall that the annihilator of a finitely generated projective module is generated by an idempotent [3] and that the exterior powers of a finitely generated projective are projective and finitely generated. (Notice: if $P \oplus Q=F$, then $\oplus \sum_{0 \leqq i \leqq p} \wedge^{i} P \otimes \bigwedge^{p-i} Q \cong \bigwedge^{p} F$.) 
Now we apply this formulation of projectivity to a number of cases where something else besides the flatness of the module is assumed. We begin with the following [8].

THEOREM 1.4. Let $M$ be a finitely generated flat $R$-module and $S$ a multiplicative set in $R$ consisting of nonzero divisors. Then if $M_{S}$ is $R_{S}$-projective, $M$ itself is projective.

Let $I$ denote one of the invariant factors of $M$. As we have seen before, $I_{S}=I \otimes R_{S}$ is the corresponding invariant factor of the $R_{S}$-module $M_{S}$. It is thus generated by an idempotent in $R_{S}$. We must show that $I$ is generated by an idempotent in $R$. It will follow from the next lemma, which shows the influence of [8]. More generally, let $M$ be a finitely generated $R$-module and $S$ as above. We say that $M$ is $S$-torsionfree if $s m=0, s \in S, m \in M$ implies $m=0$.

LEMMA 1.5. Let I be the annihilator of a finitely generated $S$-torsion-free $R$-module $M$. Then if for each prime ideal $P, I_{p}$ is either (1) or (0) and $I_{S}$ is generated by an idempotent, then I is generated by an idempotent.

Proof. Write $I_{S}=R_{S} e, e$ an idempotent. Let $J=R_{S} e \cap R ; J \supseteq I$. Since $I_{S}$ is the annihilator of $M_{S}, J M_{S}=(0)$ in $M_{S}$ which means that there is $s \in S$ such that $s J M=(0)$ in $M$. Since $M$ has no $S$-torsion, $J M=(0)$ and $J=I$. Let $L=R_{S}(1-e) \cap R$; $L \cap I=(0)$. Assume that $I+L$ is contained in some prime ideal $P . I_{P}$ must then be (0). Thus each finite set of elements of $I$ admits a common annihilator in $R-P$; $e$ being a sum $a_{1} / s_{1}+\cdots+a_{n} / s_{n}$, with $a$ 's in $I$, in particular there exists $r \in R-P$ so that $r e=0$. Thus $r \in R_{S}(1-e) \cap R=L$, a contradiction, and $I$ is a direct summand of $R$.

Similar to (1.4) is

THEOREM 1.6. Let $R$ be a commutative ring, $M$ a finitely generated $R$-module and $x$ a nonzero divisor with respect to both $R$ and $M$. Let $S$ denote the multiplicative set of all powers of $x$. If $M / x M$ and $R_{S}$ are projective over $R /(x)$ and $R_{S}$ resp., then $M$ is R-projective.

Proof. By the preceding it is enough to show that $M$ is a flat $R$-module. It is clear that we can assume $R$ to be local. $M / x M$ is then a free $R /(x)$-module. Let

$$
0 \longrightarrow K \stackrel{j}{\longrightarrow} F \stackrel{f}{\longrightarrow} M \longrightarrow 0
$$

be an exact sequence with $F$ free and $f$ a minimum epimorphism. Tensoring with $R /(x)$ we get the exact sequence

$$
0 \longrightarrow K / x K \longrightarrow F / x F \longrightarrow M / x M \longrightarrow 0
$$

with exactness on the left due to the fact that $x$ is not a zero divisor with respect to $M$. Since $f$ was chosen minimal and $M / x M$ is free, we get $K=x K$. Now localize $\left(^{*}\right)$ with respect to $S$ to get that $K_{S}$ is a direct summand of $F_{S}$. The relation $K=x K$ plus that $x$ is not a zero divisor in $R$ implies $K=K_{S}$. Call $g$ an epimorphism from 
$F_{S}$ onto $K$ with $g \cdot j_{S}=I d_{K}$. By restricting $g$ to $F$ we get that $K$ is a direct summand of $F$. This completes the proof.

It is a consequence of (1.4) that if the ring $R$ admits a multiplicative set of nonzero divisors $S$ such that $R_{S}$ is semilocal, then all finitely generated flat modules are projective. In [8] it is conjectured whether the converse is true and some cases are shown where this is so. Later we shall look at this question in rings of weak dimension one and here we point out that perhaps the result of the next section opens new possibilities. Of relevance in this matter is [13]:

COROLLARY 1.7. Let $R$ be a commutative ring; finitely generated flat modules are projective iff this is true for cyclic flat modules.

Proof. The invariant factors of a flat module being at each prime either (1) or (0) it implies for one such, say $I$, that $R / I$ is flat. The conclusion now follows from (1.3).

2. Lifting. Let $J$ be an ideal of the ring $R$ and let $M$ be a finitely generated $R$-module. Sometimes it is possible to raise the projectivity of $M / J M$ with respect to $R / J$ to that of $M$ (see [16] for not necessarily commutative rings). Here we touch the case where $M$ is $R$-flat and $J$ is the Jacobson radical of $R$.

THEOREM 2.1. Let $M$ be a finitely generated flat module and let $J$ be the Jacobson radical of $R$. If $M / J M$ is $R / J$-projective, then $M$ is $R$-projective.

Proof. Let $I=$ annihilator of $M$ and let $L=$ annihilator of $M / J M$. We claim that $L=I+J$. It is enough to verify this equation at each prime $P$ of $R$. If $M_{P}=(0)$ then $I_{P}=R_{P}=L_{P}$. If $M_{P} \neq(0)$, it is a free $R_{P}$-module and annihilator of $M_{P} / J M_{P}=J_{P}$ $=L_{P}$. As an $R / J$-module the annihilator of $M / J M$ is $I+J / J$ and by assumption and (1.3) $I+J / J$ is principal. As $I+J / J \cong I / I \cap J$, we can write $I=(a)+I \cap J$. We claim that $I=(a)$. This time we verify equality by localizing at maximal ideals; let $P$ be one such. If $(1)=I_{P}=(a)_{P}+(I \cap J)_{P}$ we must have $(a)_{P}=(1)$. If $I_{P}=(0)$, $(a)_{P}=(0)$ also and $I$ and $(a)$ are locally equal and thus $I=(a)$. To complete the proof we remind that the invariant factors are annihilators of finitely generated flat modules and that the isomorphism $\bigwedge^{p} M \otimes R / J \cong \bigwedge^{p}(M / J M)$ holds for any ideal.

3. Ideals. We begin with an application of (1.3) to ideals [15]:

COROLlaRY 3.1. Let $I$ be a finitely generated ideal of a commutative ring $R$. Then I is projective iff it is flat and its annihilator is finitely generated.

(1.3) takes this form because if $I$ is locally principal then its higher exterior powers $(p \geqq 2)$ are zero. We remark that if $J$ is the annihilator of the finitely generated flat ideal $I$, then $I \cap J=(0)$ and we can view $I$ as an ideal of $R / J$; with respect to this new ring $I$ is a projective ideal since it is still flat and its annihilator is now trivial. It is not true however that any finitely generated flat ideal is projective as the next example shows.

EXAmple 3.2. A principal flat ideal which is not projective. Let $A$ be the ring 
without identity obtained by taking a nonfinite direct sum of copies of $Z / 2 Z$, $A=\oplus \sum_{\alpha}(Z / 2 Z)_{\alpha}$ and defining addition and multiplication componentwise. Let $R$ be the ring obtained by adding the identity of $Z$ to $A: R=Z \times A$ where, we recall, addition is defined componentwise and multiplication is given by $(n, a) \cdot\left(n^{\prime}, a^{\prime}\right)$ $=\left(n n^{\prime}, n a^{\prime}+n^{\prime} a+a a^{\prime}\right)$. Let $f=(2,0)$; we observe that $f$ is not the zero element of $R$. Its annihilator is $A$ which is a not finitely generated ideal of $R$. We claim that $R f$ is a flat ideal. It is enough to show that it is free (of rank 1 or 0 ) at each prime ideal $P$. If $f \notin P$ then $(R f)_{P}=R_{P}$; if $f \in P$ but $A \notin P$, then $A_{P}=R_{P}$ and $(R f)_{P}=(0)$. Assume $R f+A \subset P$; let $g \in A$-then $g^{2}=g$ and $(1-g) g=0$. Since $1-g \notin P$, every element of $A$ is zero in $R_{P}$ and $(R f)_{P}$ is a principal ideal with trivial annihilator. This concludes the proof.

For later reference we point out another property of this ring which parallels those of an example of Nagata [14] for rings containing a common field. Let $P$ be a prime ideal of $R$; if $A \subset P, R_{P}$ is simply the ring of integers localized at some prime, i.e. $R_{P}=Z_{(P)}$ with $P \cap Z=(p)$, since the elements of $A$, as observed earlier, do not survive the localization. If however $A \notin P$, say if $e_{\alpha}$, the vector of $A$ with coordinate 1 at the $\alpha$ th place and 0 elsewhere, it is not in $P$, then all the other basic vectors of $A$ are in $P$ since for them $e_{\alpha} \cdot e_{\beta}=0$. Also $(2,0) \in P$. It is then easy to conclude that $R_{P}=Z / 2 Z$.

For some ideals however, flatness is the same as projectivity.

THEOREM 3.3. Let I be a finitely generated ideal which is a finite intersection of primary ideals. Then I is projective iff it is flat.

Proof. Write $I=I_{1} \cap \cdots \cap I_{n}$ with $I_{i} P_{i}$-primary and let $J$ be the annihilator of $I$. Say $J \subset P_{1}, \ldots, P_{r}$ but $J \notin P_{r+1}, \ldots, P_{n}$ for $0 \leqq r \leqq n$. Distinguish two cases.

(i) $J \subset$ all $P$ 's. Let $P$ be any prime ideal containing $I$. Then $P$ contains some $P_{i}$ and so also $J$. Thus $J_{P}=(0)$; if $I \notin P, I_{P}=R_{P}$. Thus $J$ is locally trivial.

(ii) $r<n$. Let $P$ be a prime ideal containing $P_{r+1}+J$ and let $j \in J-P_{r+1}$. Then $J_{P}=(0)$ means that there is $s \in R-P$ with $s j=0$ and $s \in P_{r+1}$, a contradiction. Thus $P_{r+1}$ and $J$ are comaximal. Same is true for $I_{r+1}$ and $J$ and one can even write

$$
I_{r+1} \cap \cdots \cap I_{n}+J=R .
$$

If $r=0$, we have $I+J=R$ and as $I \cap J=(0), J$ would be a direct summand of $R$. Thus assume $0<r<n$. We claim that $J \subset I_{1} \cap \cdots \cap I_{r}$. Let $j \in J-I_{1}$, say. As $J \subset P_{1}, J_{P_{1}}=(0)$ and so there is $s \in R-P_{1}$ so that $s j=0$ and since $I_{1}$ is primary, $j \in I_{1}$. $\left(^{*}\right)$ says that we can write $1=a+j_{0}$ with $a \in I_{r+1} \cap \cdots \cap I_{n}$ and $j_{0} \in J$. For $j \in J$ we have $j=j a+j j_{0}$. Since $j a \in I_{1} \cap \cdots \cap I_{n} \cap J=(0), j a=0$ and $J$ is generated by $j_{0}$.

COROLlaRY 3.4. Let $P$ be a finitely generated prime ideal. Then $P$ is projective iff it is flat. In this case either $P$ has trivial annihilator or it is a direct summand of $R$.

The last statement comes from the proof. 
If $R$ is a coherent ring then, of course, finitely generated flat ideals are projective since they are finitely related (see [6] for several characterizations of coherent rings). Here the only part of the coherency that is needed is that the annihilator of a finitely generated ideal be finitely generated. Let us call a ring with such a property precoherent (!). In this case we have

THEOREM 3.5. Let $R$ be a precoherent ring and $M$ a finitely generated flat submodule of a projective module. Then $M$ is projective.

Proof. Can assume that $M$ is a submodule of a finitely generated free module $F$. In this case the annihilator of $M$ is the annihilator of the ideal $I$ generated by all the coordinates of elements in $M$. Since $M$ is finitely generated, $I$ is finitely generated and by the precoherency of $R$ its annihilator is finitely generated. Now we must show that the higher invariant factors of $M$ are also finitely generated. In [9] it is proved that any inclusion $F_{0} \subset F$ between free modules extends to an inclusion of their exterior powers. Thus in the present situation we have the inclusion $M \subset F$ extending to an inclusion of the corresponding exterior powers since it is so by localizing at each prime of $R$. This concludes the proof.

4. Semihereditary rings. We recall that a commutative ring $R$ is said to be semihereditary if every finitely generated ideal is projective. On the other hand $R$ is said to have weak dimension $\leqq 1$ if every finitely generated ideal is flat (and so any ideal). Thus a semihereditary ring has weak dimension $\leqq 1$ and the extent to which the converse is true will be discussed here. First we glance at the prime ideals of a ring of weak dimension at most one.

THEOREM 4.1. Let $R$ be a ring of weak dimension at most one and let I be a finitely generated prime ideal. Then $I$ is either maximal or a direct summand of $R$.

Proof. By (3.4) if $I$ is not a direct summand of $R$ its annihilator is trivial. Assume this to be the case and also that $I$ is not maximal. Let $P$ be a maximal ideal containing $I$ and $x \in P-I$. Consider the exact sequences

$$
0 \longrightarrow I \longrightarrow R \longrightarrow R / I \longrightarrow 0
$$

and

$$
0 \longrightarrow R / I \stackrel{x}{\longrightarrow} R / I \longrightarrow R /(I, x) \longrightarrow 0
$$

from which we shall conclude that weak $\operatorname{dim}_{R} R /(I, x) \geqq 2$. First, by tensoring $\left(^{*}\right)$ with $R / P$ we get $\operatorname{Tor}_{1}^{R}(R / I, R / P)=I / P I$. Now this module is different from (0): by localizing at $P, I_{P}=P I_{P}$ would imply by Nakayama's lemma that $I_{P}=(0)$ which contradicts the fact that $I$ has no annihilator. On the other hand, tensoring $\left({ }^{* *}\right)$ by $R / P$ we get

and

$$
\operatorname{Tor}_{2}^{R}(R / P, R /(I, x)) \longrightarrow \operatorname{Tor}_{1}^{R}(R / P, R / I) \stackrel{x}{\longrightarrow} \operatorname{Tor}_{1}^{R}(R / P, R / I)
$$

$$
\operatorname{Tor}_{2}^{R}(R / P, R /(I, x)) \neq(0)
$$


since multiplication by $x$ induces the zero map on $I / P I$. Thus $I$ must be maximal.

The following is a characterization of semihereditary rings which should be compared to those in [7]:

THEOREM 4.2. For a commutative ring $R$ the following are equivalent:

(i) $R$ is semihereditary;

(ii) $R$ has weak dimension at most one and the annihilator of each element is finitely generated.

Proof. That (i) implies (ii) it is patent. In the other direction it will follow from the next lemma.

LEMMA 4.3. Let I and $J$ be two finitely generated projective ideals in a ring of weak dimension at most one. Then $I+J($ and $I \cap J)$ are projective ideals.

Proof. The annihilators $0: I$ and $0: J$ of $I$ and $J$ resp., are finitely generated. Since, say, $0: I$ is locally (1) or (0), $R / 0: I$ is a flat module. Thus $(0)=\operatorname{Tor}_{1}^{R}(R / 0: I, R / 0: J)$ $=(0: I) \cap(0: J) /(0: I) \cdot(0: J)$ by [5, Chapter VI, Exercise 19] and $(0: I) \cap(0: J)$ is finitely generated. As this last ideal is the annihilator of $I+J$ the conclusion follows by (3.1). The statement on $I \cap J$ is a consequence of the preceding and the usual exact sequence

$$
0 \longrightarrow I \cap J \longrightarrow I \oplus J \longrightarrow I+J \longrightarrow 0 .
$$

In (3.2) the localizations of $R$ are always discrete valuation rings or fields. Thus $R$ has weak dimension one but it is not semihereditary. The following gives an example where condition (ii) above is easily verified.

EXAMPLE 4.4. Let $k$.be a field and let $G$ be an abelian group of rank one having no element of order divisible by the characteristic of $k$. Then $k[G]$, the group algebra of $G$ over $k$, is semihereditary. That it has weak dimension one it is in [1]. Let $f$ be an element of $k[G]$ and let $H$ be a finitely generated subgroup of $G$ containing all elements which enter in the composition of $f$. Then $k[H]$ is noetherian and $I_{0}$, the annihilator of $f$ in $k[H]$, is finitely generated. Consider the exact sequence

$$
0 \longrightarrow I_{0} \longrightarrow k[H] \stackrel{f}{\longrightarrow} k[H]
$$

induced by multiplication by $f$. As $k[G]$ is a free $k[H]$-module, by tensoring the above by $k[G]$ over $k[H]$, we get that $I_{0} \cdot k[G]$ is the annihilator of $f$ in $k[G]$.

(4.3) shows that if every principal ideal in a ring of weak dimension one is projective, then the ring is semihereditary. If more flat modules are allowed to be projective we have

THEOREM 4.5. Let $R$ be a ring of weak dimension at most one. Then if every cyclic flat module is projective, $R$ is a direct sum of finitely many Prüfer domains.

Proof. By (1.7) the hypothesis implies that every finitely generated flat module is projective. Let $P_{0}$ be a minimal prime of $R$. Since for each prime $P$ of $R R_{P}$, is a 
domain, $R / P_{0}$ is a flat module. Thus $P_{0}$ is finitely generated. Since $\left(P_{0}\right)_{P_{0}}=(0), P_{0}$, by (3.4), must be a direct summand of $R$. If there were infinitely many minimal primes we could get an infinite set of orthogonal idempotents and the ideal $J$ they generate would not be finitely generated while $R / J$ would be flat. Thus there are only finitely many minimal primes and they are comaximal. By [11, p. 59] $R$ is then a finite direct sum of domains, in this case, Prüfer domains.

By (1.4) the converse holds.

A final remark: In [10] Prüfer domains are characterized as domains such that for each ideal $I, \wedge^{2} I=(0)$. This essentially follows from the fact that for $I$ finitely generated it implies $I$ projective plus that $\bigwedge^{p}$ and direct limits commute. The same condition, but for rings without nontrivial nilpotents elements, characterizes rings of weak dimension at most one. The following seems harder: Let $R$ be a domain and $I$ an ideal. Under what conditions can one ascertain that $\wedge^{2} I=(0)$ implies $I$ flat? The converse is true since flat modules are direct limits of projective modules [12] and rank considerations.

\section{REFERENCES}

1. M. Auslander, On regular group rings, Proc. Amer. Math. Soc. 8 (1957), 658-664.

2. M. Auslander and D. Buchsbaum, Invariant factors and two criteria for projectivity of modules, Trans. Amer. Math. Soc. 104 (1962), 516-522.

3. M. Auslander and O. Goldman, Maximal orders, Trans. Amer. Math. Soc. 97 (1960), $1-24$.

4. N. Bourbaki, Eléments de mathématique. Algèbre commutative, Chapters I-II, Hermann, Paris, 1961.

5. H. Cartan and S. Eilenberg, Homological algebra, Princeton Univ. Press, Princeton, N. J., 1956.

6. S. U. Chase, Direct product of modules, Trans. Amer. Math. Soc. 97 (1960), 457-473.

7. S. Endo, On semi-hereditary rings, J. Math. Soc. Japan 13 (1961), 109-119.

8. - On flat modules over commutative rings, J. Math. Soc. Japan 14 (1962), 284-291.

9. H. Flanders, Tensor and exterior powers, J. Algebra 7 (1967), 1-24.

10. C. U. Jensen, A remark on flat and projective modules, Canad. J. Math. 18 (1966), 943-949.

11. I. Kaplansky, Commutative rings, Queen Mary College Math. Notes, London, 1966.

12. D. Lazard, Sur les modules plats, C. R. Acad. Sci. Paris 258 (1964), 6313-6316.

13. K. Mount, Some remarks on Fitting's invariants, Pacific J. Math. 13 (1963), 1353-1357.

14. M. Nagata, Some remarks on prime divisors, Mem. Coll. Sci. Kyoto (Ser. A) 33 (1960), 297-299.

15. W. W. Smith, Projective ideals of finite type, Notices Amer. Math. Soc. 15 (1968), 94.

16. J. R. Strooker, Lifting projectives, Nagoya Math. J. 27 (1966), 747-751.

17. O. Zariski and P. Samuel, Commutative algebra, Vol. I, Van Nostrand, Princeton, N. J., 1958.

\footnotetext{
Rutgers, The State University, New Brunswick, NeW Jersey
} 